\title{
Diagnósticos de enfermagem prevalentes no bloco materno-infantil de um hospital público de Brasília
}

Recebido em: 14/02/2014

Aprovado em: 29/05/2014
Rinaldo de Souza Neves ${ }^{1}$

Pedro Henrique Meireles de Araújo²

Tereza Cristina Lemes Lacerda ${ }^{3}$

Resumo: Trata-se de um estudo de abordagem quantitativa que propõe a formulação dos diagnósticos de enfermagem prevalentes em cinco setores do Bloco Materno-Infantil, por meio da utilização dos diagnósticos da North American Nursing Diagnoses Association e da Classificação Internacional das Práticas de Enfermagem. Foi realizada uma análise de prontuários para identificação dos diagnósticos de enfermagem prevalentes nesses setores, adotando-se o critério de cinco diagnósticos prevalentes por setor. No total, foram mapeados 30 diagnósticos de enfermagem prevalentes, dentre estes a dor aguda, mais prevalente em mulheres gestantes no Centro Obstétrico, o risco de crescimento desproporcional em crianças internadas na Unidade de Terapia Intensiva Pediátrica, o risco de infecção em neonatos internados na Unidade de Terapia Intensiva Neonatal e em recém-nascidos e puérperas internadas no Alojamento Conjunto e o padrão respiratório ineficaz em crianças internadas na Pediatria. Os diagnósticos foram comparados e identificados semelhanças entre os resultados encontrados na literatura.

Descritores: Assistência de Enfermagem, Diagnósticos de enfermagem, Enfermagem obstétrica, Enfermagem pediátrica.

\section{Prevalent nursing diagnoses in maternal and child bloc of a public hospital in Brasilia}

Abstract: It is about a quantitative approach study which offers the formulation of the prevalent nursing diagnoses into five sectors of the Maternal and Child Bloc, through the use of the diagnosis of North American Nursing Diagnosis Association and the International Classification of Nursing Practice. A medical records analysis was performed to identify the prevalent nursing diagnoses in these sectors, adopting the criterion of five prevalent diagnostics by sector. In general, 30 prevalent nursing diagnoses were mapped, among these the sharp pain - more prevalent in pregnant women in the obstetric center -, the disproportionate growth risk for hospitalized children in Pediatric Intensive Care Unit, the contamination risk on hospitalized neonates in Neonatal Intensive Care Unit and on newborns and hospitalized postpartum women in the cooperative accommodation and the ineffective breathing standard in hospitalized children in the Pediatrics. The diagnoses were compared and similarities were found between the results obtained in the literature. Descriptors: Nursing Care, Nursing diagnoses, Obstetric nursing, Pediatric nursing.

\section{Diagnósticos de enfermería prevalentes en el bloque materno-infantil de un hospital público de Brasília}

Resumen: Se trata de un estudio de abordaje cuantitativo que propone la formulación de diagnósticos de enfermería mas frecuentes en cinco sectores del Bloque Materno-infantil, mediante el uso de los diagnósticos de la North American Nursing Diagnosis Association y de la Clasificación Internacional de la Práctica de Enfermería. Se analizaron los registros médicos para identificar los diagnósticos de enfermería mas frecuentes en estos sectores, seleccionando los cinco diagnósticos de mayor incidencia por sector. En total se mapearon los 30 diagnósticos de enfermería mas frecuentes , entre ellos el dolor agudo más frecuente en las mujeres embarazadas en el Centro Obstétrico, el riesgo de un crecimiento desproporcionado de niños ingresados en la Unidad de Cuidados Intensivos Pediátricos, el riesgo de infección en los recién nacidos en la Unidad de Cuidados Intensivos Neonatales y del recién nacido y pos-parto hospitalizados en el Alojamiento Conjunto y el patrón respiratorio ineficaz en niños hospitalizados en Pediatría. Los diagnósticos fueron comparados y se identificó similitudes con los resultados encontrados en la literatura.

Descriptores: Cuidados de Enfermería, Los diagnósticos de enfermería, Enfermería Neonatal, Enfermería pediátrica.

\section{INTRODUÇÃO}

$\mathrm{O}$ Processo de Enfermagem (PE) é baseado em princípios e regras que são conhecidos por promover cuidado de enfermagem eficiente. É definido como "a dinâmica das ações sistematizadas e inter-relacionadas, visando à assistência ao ser humano"(1). Pode ser denominado, ainda, de Sistematização da Assistência de Enfermagem (SAE) ou Metodologia da Assistência de Enfermagem (MAE)(1).

As NHBs foram hierarquizadas por Maslow em cinco níveis: necessidades fisiológicas, de segurança, de amor, de estima e de autorrealização. Um indivíduo só procura satisfazer as necessidades do nível seguinte após um mínimo de satisfação das anteriores. $\mathrm{O}$ mínimo referido ainda não foi determinado, mas o próprio autor reconhece que tal sistemática não é rígida, variando também em alguns indivíduos(2).

Dentre as etapas da SAE, o Histórico de Enfermagem é o roteiro sistematizado para o levantamento de dados significativos (para o enfermeiro) do ser humano, os quais tornam possível a identificação de seus problemas ${ }^{(2)}$.
A Classificação Internacional para a Prática de Enfermagem (CIPE) é um instrumento de informação para descrever a prática de enfermagem, prover dados que identifiquem a contribuição da enfermagem no cuidado de saúde e promover mudanças por meio da educação, pesquisa e administração. Tem como objetivos estabelecer uma linguagem comum que descreva a prática de enfermagem; descrever mundialmente a prestação do cuidado de enfermagem; possibilitar a comparação dos dados de enfermagem entre diferentes populações de pacientes, locais de atendimento, tempo, dentre outros objetivos ${ }^{(1)}$.

$\mathrm{Na}$ Enfermagem brasileira, os vocabulários mais conhecidos e utilizados na prática profissional são os diagnósticos de enfermagem da North American Nursing Diagnoses Association-Internacional (NANDA-l), as intervenções da North Intervention Classification (NIC), os resultados de enfermagem da North Outcomes Classification (NOC) e a Classificação CIPE.

A NANDA-I é outra classificação utilizada na Enfermagem. Sua estrutura atual conta com sete eixos (conceito, sujeito, julgamento, localização, idade, tempo e situação do diagnóstico). É multiaxial 
e organizada com 217 diagnósticos em 13 domínios e 47 classes, sendo o sistema de classificação mais utilizado no mundo. Tem como componentes estruturais: enunciado, fatores relacionados, características definidoras, fatores de risco e definição do diagnóstico. A NANDA-I classifica, além dos diagnósticos reais, os diagnósticos de risco, de bem-estar e de promoção da saúde ${ }^{(3)}$.

A CIPE é a classificação multiaxial constituída por sete eixos: foco (área de atenção relevante para a enfermagem); juízo (opinião clínica relacionada com o foco); meios (forma ou método de concretizar uma intervenção); ação (processo intencional aplicado a, ou desempenhado por, um paciente); tempo (o período, intervalo ou duração de uma ocorrência); localização (orientação anatômica ou espacial de um diagnóstico ou intervenção); e paciente (sujeito a quem o diagnóstico se refere). Serve tanto para construir diagnósticos quanto para construir intervenções, fazendo uso de termos do eixo de ação e de foco(1).

A NOC é a taxonomia complementar às taxonomias da NANDA e da NIC. Ela determina a condição da saúde de um paciente, cuidador, família ou comunidade, com a finalidade de observar as mudanças apresentadas por eles após as intervenções de enfermagem. Possui 330 resultados, cada um constituído por um nome identificador, uma definição, uma lista de indicadores para se determinar a condição de saúde do indivíduo/família/comunidade em relação ao resultado avaliado, uma escala de medidas de 5 pontos e uma lista com as referências bibliográficas ${ }^{(1)}$.

Após elaborar os diagnósticos de enfermagem, o enfermeiro deverá buscar na taxonomia NOC o resultado mais adequado para o paciente. Seu uso se faz importante pelo fato de ser essencial que cada disciplina identifique os resultados que tenham sido influenciados por sua prática, assegurando que sejam incluídos na avaliação da eficiência dos cuidados de saúde ${ }^{(1)}$.

Para auxiliar na etapa da implementação da assistência de Enfermagem, o enfermeiro pode consultar a NIC, que é uma taxonomia de intervenções de enfermagem criada pela necessidade de padronizar a linguagem usada pelos enfermeiros na descrição dos cuidados que eles realizavam com os pacientes ${ }^{(4)}$.

Diante da necessidade de criar uma ferramenta de apoio para o processo de trabalho dos enfermeiros no cenário do estudo, levantou-se a seguinte questão norteadora: quais os diagnósticos de enfermagem prioritários no Bloco Materno-Infantil do Hospital Materno-Infantil de Brasília (HMIB)?

Este estudo tem como objetivo geral definir a prevalência dos diagnósticos de enfermagem no Centro Obstétrico (CO), na Unidade de Terapia Intensiva Pediátrica (UTIp), na Unidade de Terapia Intensiva Neonatal (UTIn), no Alojamento Conjunto (Alcon) e na Unidade de Pediatria do HMIB, da Secretaria de Estado de Saúde do Distrito Federal (SES-DF).

\section{METODOLOGIA}

Este é um estudo de abordagem quantitativa, em que se realizou análise de prontuários, com o objetivo de definir a prevalência dos diagnósticos de enfermagem em seus respectivos setores. Adotou-se o critério de escolha de cinco diagnósticos de enfermagem prevalentes para os setores em estudo.

Para a determinação da prevalência dos diagnósticos de enfermagem em gestantes no CO, puérperas no Alcon/Maternidade e crianças na Pediatria, UTIn e UTIp, foram utilizadas diferentes procedimentos para a coleta de dados.

Para os setores de UTIn e UTIp, realizou-se análise de prontuário pelo InterSystems TrakCare ${ }^{\circledR}$. Foram avaliadas as evoluções de enfermagem e médica para mapeamento dos diagnósticos de enfermagem e definição de sua prevalência nesse setor.

Nos setores de Pediatria, Alcon e CO, os dados foram coletados no prontuário eletrônico por meio do registro de evoluções dos internos de enfermagem da ESCS no InterSystems TrakCare ${ }^{\circledR}$.

Optou-se pela escolha de cinco diagnósticos de enfermagem prioritários para a gestante no $\mathrm{CO}$, cinco para a puérpera no Alcon, cinco para o recém-nascido (RN) no Alcon, cinco para o RN em UTIn, cinco para a criança em Pediatria, cinco para a criança em UTI pediátrica, em um total de 30 diagnósticos, identificados por meio da análise de prontuários no InterSystems TrakCare ${ }^{\circledast}$.

Foram incluídos os prontuários que continham os registros de enfermagem dos internos do curso de Enfermagem da ESCS e excluídos os prontuários que não apresentavam as evoluções de enfermagem.

O projeto foi aprovado pelo Comitê de Ética em Pesquisa (CEP) da Fundação de Ensino e Pesquisa em Ciências da Saúde (FEPECS) sob o número de protocolo $278 / 12$.

\section{RESULTADOS E DISCUSSÃO}

Por meio da metodologia de análise dos prontuários, foram identificados os diagnósticos de enfermagem prevalentes em gestantes no CO, crianças na UTIp, UTIn e Pediatria, RN no Alcon e puérperas no Alcon.

\section{Diagnósticos de Enfermagem Prevalentes no Centro Obstétrico}

Do total de 25 prontuários de pacientes internadas no $\mathrm{CO}$, encontrou-se a seguinte ordem de prevalência, conforme mostra a Tabela 1:

Tabela 1 - Distribuição dos Diagnósticos de Enfermagem documentados nos prontuários de mulheres gestantes no Centro Obstétrico do HMIB. Brasília - DF, dezembro, 2013.

\begin{tabular}{lll}
\hline $\begin{array}{l}\text { Diagnósticos de Enfermagem } \\
\text { (NANDA-I, 2012-2014) }(\mathbf{N}=\mathbf{2 5})\end{array}$ & $\mathbf{n}$ & $\%$ \\
\hline 1. Dor aguda & 10 & 40 \\
\hline 2. Risco de infecção & 8 & 32 \\
\hline 3. Ansiedade & 7 & 28 \\
\hline 4. Risco de glicemia instável & 6 & 24 \\
$\begin{array}{l}\text { 5. Risco de díade mãe/feto } \\
\text { perturbada }\end{array}$ & 5 & 20 \\
\hline
\end{tabular}

Dos diagnósticos de enfermagem identificados nesta pesquisa e apresentados na Tabela 1, dois diagnósticos também foram encontrados e corroboram os estudos de Sumita ${ }^{(5)}$ no CO do hospital de São Paulo, que foram: risco para infecção (100\%) e dor aguda $(90,6 \%)$. Diferentemente desses estudos, quatro diagnósticos não foram identificados nesta pesquisa: risco para perfusão tissular materno-fetal ineficaz, conforto alterado, nutrição desequilibrada para menos que as necessidades corporais e medo.

\section{Diagnósticos de Enfermagem Prevalentes na UTI Pediátrica}

Do total de 20 prontuários de crianças internadas na UTI pediátrica, identificou-se a seguinte ordem de prevalência, conforme mostra a Tabela 2: 
Tabela 2 - Distribuição dos Diagnósticos de Enfermagem documentados nos prontuários de crianças internadas na UTI Pediátrica do HMIB. Brasília - DF, dezembro, 2013.

\begin{tabular}{|lcc|}
\hline $\begin{array}{l}\text { Diagnósticos de Enfermagem (NANDA-I, } \\
\text { 2012-2014) (N = 20) }\end{array}$ & $\mathbf{n}$ & $\%$ \\
\hline 1. Risco de crescimento desproporcional & 20 & 100 \\
\hline 2. Risco de atraso no desenvolvimento & 20 & 100 \\
\hline 3. Risco de infecção & 18 & 90 \\
\hline 4. Desobstrução ineficaz das vias aéreas & 14 & 70 \\
\hline 5. Deglutição prejudicada & 14 & 70 \\
\hline
\end{tabular}

Percebe-se que os diagnósticos de enfermagem identificados nessa amostra são distintos quando comparados com a pesquisa de Maia, Neves e Souza ${ }^{(6)}$ em um hospital público de Brasília, pois o estudo identificou cinco diagnósticos de enfermagem com frequência acima de $60 \%$ na amostra, que foram: integridade da pele prejudicada, nutrição desequilibrada para menos do que as necessidades corporais, troca de gases prejudicada, risco de glicemia instável e ventilação espontânea prejudicada.

\section{Diagnósticos de Enfermagem Prevalentes na UTI Neonatal}

Do total de 20 prontuários de neonatos internados na UTI neonatal, identificou-se a seguinte ordem de prevalência, conforme mostra a Tabela 3:

Tabela 3 - Distribuição dos Diagnósticos de Enfermagem documentados nos prontuários de neonatos internados na UTI Neonatal do HMIB. Brasília - DF, dezembro, 2013.

\begin{tabular}{|lcc|}
\hline $\begin{array}{l}\text { Diagnósticos de Enfermagem (NANDA-I, } \\
\text { 2012-2014) (N= 20) }\end{array}$ & $\mathbf{n}$ & $\%$ \\
\hline $\begin{array}{l}\text { 1. Risco de infecção } \\
\text { 2. Amamentação interrompida }\end{array}$ & 20 & 100 \\
\hline 3. Risco de atraso no desenvolvimento & 20 & 100 \\
\hline 4. Processos familiares interrompidos & 19 & 95 \\
\hline 5. Integridade da pele prejudicada & 18 & 90 \\
\hline
\end{tabular}

A Tabela 3 apresenta dois diagnósticos de enfermagem prevalentes e semelhantes que coincidem com os estudos de $\mathrm{Melo}^{(7)}$, sendo eles: integridade da pele prejudicada e risco para redução da integridade da pele; risco de infecção. A autora ainda identificou quatro diagnósticos de enfermagem: risco para hipotermia, risco para hipertermia, risco para padrão ineficiente da respiração e risco para injúria do sistema nervoso central ${ }^{(7)}$. Diferentemente desse estudo, uma pesquisa ${ }^{(8)} \mathrm{com}$ neonatos mostrou dois diagnósticos de enfermagem com frequência igual a $100 \%$ que foram: risco de choque e risco de desequilíbrio de volume de líquidos. A frequência do diagnóstico risco de atraso no desenvolvimento para esse mesmo estudo foi menor, quando comparado a essa pesquisa realizada no HMIB.

\section{Diagnósticos de Enfermagem Prevalentes no Alcon - RN}

Do total de 20 prontuários de RNs internados no Alcon, encontrou-se a seguinte ordem de prevalência, conforme mostra a Tabela 4:
Tabela 4 - Distribuição dos Diagnósticos de Enfermagem documentados nos prontuários de recém-nascidos internados no Alcon do HMIB. Brasília - DF, dezembro, 2013.

\begin{tabular}{lll}
\hline $\begin{array}{l}\text { Diagnósticos de Enfermagem (NANDA-I, } \\
\text { 2012-2014) (N=20) }\end{array}$ & $\mathbf{n}$ & $\%$ \\
\hline $\begin{array}{l}\text { 1. Risco de infecção } \\
\text { 2. Amamentação eficaz }\end{array}$ & 18 & 90 \\
\hline $\begin{array}{l}\text { 3. Risco de desequilíbrio na temperatura } \\
\text { corporal }\end{array}$ & 14 & 70 \\
\hline 4. Risco de glicemia instável & 9 & 45 \\
\hline 5. Icterícia neonatal & 4 & 20 \\
\hline
\end{tabular}

A Tabela 4 mostra que dois diagnósticos de enfermagem apresentaram maior frequência, ou seja, o diagnóstico de risco de infecção (90\%) e amamentação eficaz (70\%). Estudo ${ }^{(9)}$ realizado em 35 neonatos também mostrou frequência elevada dos diagnósticos de enfermagem amamentação eficaz $(88,5 \%)$ e risco para infecção (100\%), o que corresponde aos dois diagnósticos identificados nesse estudo. Os autores também formularam o diagnóstico de enfermagem risco de desequilíbrio na temperatura corporal (100\%) da amostra, o que difere dos resultados apresentados na Tabela 4.

\section{Diagnósticos de Enfermagem Prevalentes no Alcon - Puérpera}

Do total de 20 puérperas internadas no Alcon, identificou-se a seguinte ordem de prevalência, conforme mostra a Tabela 5:

Tabela 5 - Distribuição dos Diagnósticos de Enfermagem documentados nos prontuários de puérperas internadas no Alcon do HMIB. Brasília - DF, dezembro, 2013.

\begin{tabular}{lll}
\hline $\begin{array}{l}\text { Diagnósticos de Enfermagem (NANDA-I, } \\
\text { 2012-2014) (N= 20) }\end{array}$ & $\mathbf{n}$ & $\%$ \\
\hline $\begin{array}{l}\text { 1. Risco de infecção } \\
\text { 2. Risco de sangramento }\end{array}$ & 19 & 95 \\
\hline 3. Integridade da pele prejudicada & 14 & 70 \\
\hline 4. Amamentação eficaz & 11 & 55 \\
\hline 5. Conforto prejudicado & 4 & 40 \\
\hline
\end{tabular}

O diagnóstico de enfermagem risco de infecção esteve presente em $95 \%$ dos prontuários de puérperas no HMIB. Esse resultado também se confirma para Patine e Furlan ${ }^{(10)}$, que apresentou prevalência igual a 93,5\% em unidade de ginecologia e obstetrícia. Entretanto, o diagnóstico integridade da pele prejudicada apresentou prevalência de $70 \%$ nesse estudo e o diagnóstico integridade tissular prejudicada mostrou resultado igual a $74,2 \%$ para Patine e Furlan ${ }^{(10)}$. Nesse sentido, um estudo ${ }^{(11)}$ revela que a maioria das puérperas apresenta risco para infecção localizada nos sítios das mamas, da vagina, do abdome e do períneo.

\section{Diagnósticos de Enfermagem Prevalentes na Pediatria}

Do total de 20 prontuários de crianças internados na Ala A e $B$ da Pediatria, identificou-se a seguinte ordem de prevalência, conforme mostra a Tabela 6: 
Tabela 6 - Distribuição dos Diagnósticos de Enfermagem documentados nos prontuários de crianças internadas na Pediatria do HMIB. Brasília - DF, dezembro, 2013.

\begin{tabular}{lll}
\hline $\begin{array}{l}\text { Diagnósticos de Enfermagem (NANDA-I, } \\
\text { 2012-2014) (N=20) }\end{array}$ & $\mathbf{n}$ & $\%$ \\
\hline $\begin{array}{l}\text { 1. Padrão respiratório ineficaz } \\
\text { 2. Risco de infecção }\end{array}$ & 9 & 45 \\
\hline 3. Desobstrução ineficaz das vias aéreas & 5 & 40 \\
\hline 4. Troca de gases prejudicada & 5 & 25 \\
\hline 5. Débito cardíaco diminuído & 3 & 25 \\
\hline
\end{tabular}

A Tabela 6 mostra a frequência dos diagnósticos de enfermagem de padrão respiratório ineficaz (45\%) e risco de infecção (40\%) nos prontuários das crianças internadas na Pediatria. Esse resultado também foi encontrado no estudo realizado em uma unidade de Pediatria hospitalar ${ }^{(12)}$, em que se identificou a frequência de $100 \%$ para o diagnóstico desobstrução ineficaz das vias áreas, $80 \%$ para o diagnóstico padrão respiratório ineficaz e $66,7 \%$ para o diagnóstico risco de infecção. Outra pesquisa ${ }^{(13)}$ corrobora com esse estudo, onde se identificou frequência diminuída do diagnóstico de enfermagem desobstrução ineficaz de vias aéreas em 151 crianças internadas em um hospital público pediátrico, que corresponde a apenas $37,7 \%$ da amostra.

\section{CONCLUSÃO}

Diante deste dinâmico processo de trabalho e da necessidade de realizar um atendimento individualizado e humanizado, a profissão requer tecnologias que auxiliem na SAE/PE para favorecer o cuidado e o trabalho do profissional. Para tal, foram realizados levantamento de dados e avaliação dos prontuários de pacientes do Bloco Materno-Infantil do HMIB e elencados diagnósticos e intervenções prioritários de enfermagem, com o objetivo de permitir a melhor atuação do enfermeiro, utilizando a Teoria das $\mathrm{NHBs}$, dos diagnósticos da NANDA e da CIPE e das intervenções de enfermagem da NIC.

A SAE organiza o trabalho do enfermeiro de maneira resolutiva e eficaz e, mas para que seja realizada com sucesso, é necessário o empenho e dedicação do enfermeiro na valorização de seu trabalho, raciocínio clínico e intervenções, como também na continuidade ao cuidado de modo a acompanhar a evolução e recuperação do paciente.

O perfil de diagnósticos de enfermagem prevalentes no Bloco Materno-Infantil, juntamente com o mapeamento bibliográfico, possibilitará a criação de uma proposta para documentação eletrônica do $\mathrm{PE}$, com os principais diagnósticos e intervenções de enfermagem para a gestante, a puérpera, o RN e a criança.

Como limitação do estudo, pode-se apontar a amostra pequena de prontuários para determinar os diagnósticos de enfermagem prevalentes nas áreas adotadas para o estudo. Além disso, foi utilizada a fonte documental para a formulação dos diagnósticos. Identificaram-se, também, dificuldades relacionadas com a participação dos supervisores de enfermagem das unidades.

Como fortaleza do projeto e da proposta, aponta-se a adesão dos estudantes de enfermagem que participaram deste estudo. Recomenda-se, portanto, a continuidade do processo de implantação do PE e utilização do sistema de informação como ferramenta para facilitação do mesmo

\section{Referências}

1. Pinheiro AM, Tannure MC. SAE: Sistematização da Assistência de enfermagem. 2. ed. Rio de Janeiro: Guanabara Koogan; 2010.

2. Horta WA. Processo de Enfermagem. Rio de Janeiro: Guanabara Koogan; 2011.

3. Nanda. Diagnósticos de enfermagem da NANDA International: definições e classificação 2012/2014. Porto Alegre: Artmed; 2013.

4. Bulechek GM, Mccloskey JC. Classificação das Intervenções de Enfermagem (NIC). 3. ed. São Paulo: Artmed; 2004.

5. Sumita SLN. O processo de enfermagem na assistência à parturiente: diagnósticos e intervenções. São Paulo: [s. n.]; 2002.

6. Maia VA, Neves MGC, Souza LM. Padrão Mínimo de Enfermagem: proposta de protocolo em uma UTI pediátrica pública do DF [Trabalho de Conclusão de Curso]. Brasília: Hospital Materno-Infantil de Brasília; 2012.

7. Melo MIS. Diagnósticos de enfermagem e propostas de intervenções em recém-nascidos pré-termo (Idade gestacional menor que 37 semanas) em Unidade de Terapia Intensiva Neonatal. Hospital Materno-Infantil de Brasília, Brasília; 2002. 8. França DF, et al. Diagnósticos de enfermagem de recém-nascidos com sepse em uma Unidade de Terapia Intensiva Neonatal. Rev. Latino-Am. Enfermagem. mar.-abr. 2014;22(2):255-61.
9. Alves, AR, et al. Diagnósticos de enfermagem em unidades de alojamento conjunto. Rev.Bras.Enferm. Brasília 2010 nov-dez: 849-9. 10. Furlan MFFM, Patine FS. Diagnósticos de enfermagem no atendimento a puérperas e recém-nascidos internados em alojamento conjunto. Arq. Ciênc. Saúde [acesso em 12 abr 2013]. 2006 out-dez;13(4):202-8. Disponível em: http://www. cienciasdasaude.famerp.br/racs_ol/vol-13-4/Famerp\%20 13\%284\%29\%20ID\%20169\%20-\%2015.pdf.

11. Bachion MM, Munari DB, Salge AKM, Vieira F. Diagnósticos de enfermagem da NANDA no período pós-parto imediato e tardio. Esc. Anna Nery [Internet]. 2010 [cited 2012 jun 30];14(1):83-9. Available from: http://dx.doi.org/10.1590/S141481452010000100013.

12. Chagas KLMC, Lima LHO, Luz GOA, Oliveira EAR. Diagnósticos de enfermagem em crianças com sinais e sintomas respiratórios: um estudo descritivo. Rev Rene. Fortaleza, 2011 abr-jun;12(2):302-8.

13. Andrade, LZC, et al. Desobstrução ineficaz de vias áreas em crianças com infecção respiratória. Rev.Eletr.Enferm. 2014. Jan. mar;16 (1):21-7. 